\title{
A NOTE ON THE BOUNDEDNESS OF SOLUTIONS OF LINEAR PARABOLIC EQUATIONS
}

\author{
A. MCNABB
}

Hartman and Wintner [1] obtained a Sturmian comparison theorem for self-adjoint second order elliptic equations of the form

$$
\sum_{i, j=1}^{n} \frac{\partial}{\partial x_{i}}\left(a_{i j} \frac{\partial u}{\partial x_{j}}\right)+f u=0, \quad a_{i j}=a_{j i}
$$

in a bounded domain $B$ with boundary $\partial B$. In this note, their method is slightly modified to prove the following theorem.

Denote by $D$ the semi-infinite cylinder $\{(x, t): x \in B, t>0\}$, by $\bar{D}$ its closure and by $D_{T}$ the intersection of $D$ with the half-space $t \leqq T$. Suppose $u=u(x)$ is a solution of equation (1) which is continuous in $\bar{B}$ the closure of $B$, vanishes on $\partial B$ and has continuous second derivatives in $B$. Again, suppose $w=w(x, t)$ is defined and continuous in the closed region $\bar{D}_{T}$, is positive on $B$ at $t=0$ and on $\partial B$ for all $t \geqq 0$ and has continuous derivatives $\partial w / \partial t, \partial^{2} w / \partial x_{i} \partial x_{j}$ which satisfy the parabolic equation

$$
\sum_{i, j=1}^{n} \frac{\partial}{\partial x_{i}}\left(A_{i j} \frac{\partial w}{\partial x_{j}}\right)+F w=C \frac{\partial w}{\partial t}, \quad A_{i j}=A_{j i},
$$

in $D_{T}$ for all $T>0$. The functions $A_{i j}, \partial A_{i j} / \partial x_{i}, F$ and $C$ are uniformly bounded continuous functions of $x_{i}$ and $t$ in $D_{T}$ for any given $T>0$, while $C$ is bounded in $D$ by two positive constants $C_{0}$ and $C_{1}$ $\left(0<C_{0} \leqq C \leqq C_{1}\right)$ and the quadratic form $\sum_{i, j} A_{i j} \xi_{i} \xi_{j}$ is non-negative at all points in $D$.

THEOREM 1. If $\int_{B}\left\{u^{2}(F-f)+\sum_{i, j=1}^{n}\left(a_{i j}-A_{i j}\right)\left(\partial u / \partial x_{i}\right)\left(\partial u / \partial x_{j}\right)\right\} d \tau$ $\geqq \epsilon(t)$ for all $t>0$ and $\int_{0}^{T} \epsilon(t) d t$ tends to infinity with $T$, then $w$ is unbounded in $D$.

Proof. Since $w>0$ on $\bar{B}$ at $t=0$ and on $\partial B$ for all $t>0$, the maximum principle for parabolic equations (see [2]) implies $w>0$ in $D_{T}$ for all $T>0$.

The Green identity leads from the boundary condition $u=0$ on $\partial B$, to the divergence relation

$$
\int_{B}\left\{\sum_{i=1}^{n} \frac{\partial}{\partial x_{i}}\left(u^{2} h^{i}\right)\right\} d \tau=0 \quad \text { for all } t \geqq 0,
$$
1961.

Received by the editors November 14, 1960 and, in revised form, February 20, 
where $h^{i}$ is the $t$-dependent vector function

$$
h^{i}=\sum_{j=1}^{n} \frac{A_{i j}}{w} \frac{\partial w}{\partial x_{j}} .
$$

This vector is finite at each point of $D$ since $w>0$ in this region.

Since $u$ is a solution of (1) and vanishes on $\partial B$, another application of Green's identity shows that

$$
\int_{B}\left\{\sum_{i, j=1}^{n} a_{i j} \frac{\partial u}{\partial x_{i}} \frac{\partial u}{\partial x_{j}}-f u^{2}\right\} d \tau=0 .
$$

It is clear from (4) and (2) that

$$
\sum_{i=1}^{n} \frac{\partial h^{i}}{\partial x_{i}}=\frac{C}{w} \frac{\partial w}{\partial t}-F-\sum_{i, j=1}^{n} \frac{A_{i j}}{w^{2}} \frac{\partial w}{\partial x_{i}} \frac{\partial w}{\partial x_{j}}
$$

and using this in conjunction with (3) and (5), we see that

$$
\begin{aligned}
\int_{B} \frac{u^{2} C}{w} \frac{\partial w}{\partial t} d \tau=\int_{B}\left\{\sum_{i, j=1}^{n}\right. & \left(a_{i j} \frac{\partial u}{\partial x_{i}} \frac{\partial u}{\partial x_{j}}-2 \frac{u}{w} A_{i j} \frac{\partial u}{\partial x_{i}} \frac{\partial w}{\partial x_{j}}\right. \\
& \left.\left.+\frac{u^{2}}{w^{2}} A_{i j} \frac{\partial w}{\partial x_{i}} \frac{\partial w}{\partial x_{j}}\right)+u^{2}(F-f)\right\} d \tau
\end{aligned}
$$

for all $t \geqq 0$. The right-hand side of this equation can be rewritten in the form

$$
\begin{aligned}
\int_{B}\left\{\sum_{i, j=1}^{n} A_{i j}\right. & \left.\left(\frac{\partial u}{\partial x_{i}}-\frac{u}{w} \frac{\partial w}{\partial x_{i}}\right)\left(\frac{\partial u}{\partial x_{j}}-\frac{u}{w} \frac{\partial w}{\partial x_{j}}\right)\right\} d \tau \\
& +\int_{B}\left\{u^{2}(F-f)+\sum_{i, j=1}^{n}\left(a_{i j}-A_{i j}\right) \frac{\partial u}{\partial x_{i}} \frac{\partial u}{\partial x_{j}}\right\} d \tau
\end{aligned}
$$

since $A_{i j}=A_{j i}$.

Since the quadratic form $\sum_{i, j=1}^{n} A_{i j} \xi_{i} \xi_{j}$ is positive definite or at least non-negative and the second term of expression (8) is assumed greater than or equal to $\epsilon(t)$, we see from (7) that $\partial z / \partial t \geqq \epsilon(t)$, where $z=\int_{B} C u^{2} \log w d \tau$. But then $z(T) \geqq z(0)+\int_{0}^{T} \epsilon(t) d t$ and is unbounded if $\int_{0}^{T} \epsilon(t) d t$ tends to infinity with $T$. If $z$ is unbounded, $w$ cannot be bounded; for if it were bounded by $w_{0}$ for all $T$ we would have $z(T) \leqq \int_{B} C_{1} u^{2} \log w_{0} d \tau$ always.

Protter [3] extended the comparison theorem of Hartman and Wintner to a form valid for a pair of general linear elliptic equations. A corresponding extension of Theorem 1 is given below.

THEOREM 2. If $\boldsymbol{a}$ and $\boldsymbol{w}$ satisfy the general linear equations 


$$
\begin{aligned}
& \sum_{i, j=1}^{n} \frac{\partial}{\partial x_{i}}\left(a_{i j} \frac{\partial u}{\partial x_{j}}\right)+\sum_{i=1}^{n} b_{i} \frac{\partial u}{\partial x_{i}}+f u=0 \\
& \sum_{i, j=1}^{n} \frac{\partial}{\partial x_{i}}\left(A_{i j} \frac{\partial w}{\partial x_{j}}\right)+\sum_{i=1}^{n} B_{i} \frac{\partial w}{\partial x_{i}}+F w=C \frac{\partial w}{\partial t}
\end{aligned}
$$

in $B$ and $D$, and the boundary and continuity conditions of Theorem 1, then $w$ is unbounded in $D$ if $\int_{0}^{T} \epsilon(t) d t$ tends to infinity with $T$ and

$$
\begin{aligned}
\int_{B}\left\{\sum_{i, j=1}^{n}\left(a_{i j}-A_{i j}\right) \frac{\partial u}{\partial x_{i}} \frac{\partial u}{\partial x_{j}}\right. & \\
+ & \left.\frac{u^{2}}{4}\left[4(F-f)-\sum_{i, j=1}^{n} A^{i j} B_{i} B_{j}+2 \sum_{i=1}^{n}\left(\frac{\partial b_{i}}{\partial x_{i}}-\frac{\partial B_{i}}{\partial x_{i}}\right)\right]\right\} d \tau
\end{aligned}
$$

is greater than or equal to $\epsilon(t)$ for all $t>0 . A^{i j}$ denote the elements of the inverse matrix of $A_{i j}$ and the functions $\partial b_{i} / \partial x_{i}, \partial B_{i} / \partial x_{i}$ are assumed continuous in $\bar{B}$ and $\bar{D}$ respectively.

Proof. Let us construct an integral corresponding to (7) with its left-hand side, like (8), consisting of an integral of a non-negative form containing all terms involving the function $w$, and a residual integral containing only terms involving the function $u$ and the coefficients of equations (9) and (10).

Consider the expression

$$
\begin{aligned}
E= & \int_{B}\left\{\sum_{i, j=1}^{n}\left(a_{i j}-A_{i j}\right) \frac{\partial u}{\partial x_{i}} \frac{\partial u}{\partial x_{j}}\right. \\
& \left.+\sum_{i=1}^{n} u \frac{\partial u}{\partial x_{i}}\left(B_{i}-b_{i}\right)+(F-f) u^{2}\right\} d \tau \\
& +\int_{B}\left\{\sum_{i, j=1}^{n} A_{i j} \frac{\partial u}{\partial x_{i}} \frac{\partial u}{\partial x_{j}}-\sum_{i=1}^{n} u \frac{\partial u}{\partial x_{i}} B_{i}-F u^{2}\right\} d \tau
\end{aligned}
$$

The term $F u^{2}$ of the second integral can be eliminated by subtracting from it the vanishing integral

$$
\begin{aligned}
E^{\prime}= & \int_{B}\left\{\sum_{i=1}^{n} \frac{\partial}{\partial x_{i}}\left(u^{2} \sum_{j=1}^{n} \frac{A_{i j}}{w} \frac{\partial w}{\partial x_{j}}\right)\right\} d \tau \\
= & \int_{B}\left\{\frac{C u^{2}}{w} \frac{\partial w}{\partial t}-F u^{2}-\frac{u^{2}}{w} \sum_{i=1}^{n} B_{i} \frac{\partial w}{\partial x_{i}}\right. \\
& \left.-\sum_{i, j=1}^{n}\left(\frac{u^{2}}{w^{2}} A_{i j} \frac{\partial w}{\partial x_{i}} \frac{\partial w}{\partial x_{j}}-2 \frac{u}{w} A_{i j} \frac{\partial u}{\partial x_{i}} \frac{\partial w}{\partial x_{j}}\right)\right\} d \tau .
\end{aligned}
$$


Since $E$ is zero by virtue of (9),

$$
\begin{aligned}
\frac{\partial z}{\partial t}= & \int_{B} \frac{C u^{2}}{w} \frac{\partial w}{\partial t} d \tau \\
= & \int_{B}\left\{\sum_{i, j=1}^{n}\left(a_{i j}-A_{i j}\right) \frac{\partial u}{\partial x_{i}} \frac{\partial u}{\partial x_{j}}\right. \\
& \left.+\sum_{i=1}^{n} u \frac{\partial u}{\partial x_{i}}\left(B_{i}-b_{i}\right)+(F-f) u^{2}\right\} d \tau \\
& +\int_{B}\left\{\sum_{i, j=1}^{n} A_{i j}\left(\frac{\partial u}{\partial x_{i}}-\frac{u}{w} \frac{\partial w}{\partial x_{i}}\right)\left(\frac{\partial u}{\partial x_{j}}-\frac{u}{w} \frac{\partial w}{\partial x_{j}}\right)\right. \\
& \left.-\sum_{i=1}^{n} u B_{i}\left(\frac{\partial u}{\partial x_{i}}-\frac{u}{w} \frac{\partial w}{\partial x_{i}}\right)\right\} d \tau \\
= & \int_{B}\left\{\sum_{i, j=1}^{n}\left(a_{i j}-A_{i j}\right) \frac{\partial u}{\partial x_{i}} \frac{\partial u}{\partial x_{j}}\right. \\
& +\int_{B}\left\{\sum _ { i , j = 1 } ^ { n } \left[4(F-f)-A_{i j}\left(\frac{\partial u}{\partial x_{i}}-\frac{u}{w} \frac{\partial w}{\partial x_{i}}-\frac{u}{2} \sum_{k=1}^{n} A_{i}^{i k} B_{k}\right)\right.\right. \\
& \left.+\left(\frac{\partial u}{\partial x_{j}}-\frac{u}{w} \frac{\partial w}{\partial x_{j}}-\frac{u}{2} \sum_{k=1}^{n} A_{i=1}^{n}\left(\frac{\partial b_{i}}{\partial x_{i}}-\frac{\partial B_{i}}{\partial x_{i}}\right)\right]\right\} d \tau
\end{aligned}
$$

where $A^{i j}$ is the inverse matrix of $A_{i j}$. The matrix $A_{i j}$ is required to be nonsingular in Theorem 2 as well as non-negative in $D$. Since $\sum_{i, j=1}^{n} A_{i j} \xi_{i} \xi_{j}$ is positive definite in $D$ the second integral of (14) is non-negative. The remainder of the proof now proceeds as for Theorem 1.

\section{BIBLIOGRAPHY}

1. P. Hartman and A. Wintner, On a comparison theorem for self-adjoint partial differential equations of elliptic type, Proc. Amer. Math. Soc. 6 (1955), 862.

2. L. Nirenberg, A strong maximum principle for parabolic equations, Comm. Pure Appl. Math. 6 (1953), 167-177.

3. M. H. Protter, A comparison theorem for elliptic equations, Proc. Amer. Math. Soc. 10 (1959), 296.

Applied Mathematics Laboratory,

DEPARTMENT OF SCIENTIFIC AND INDUSTRIAL Research, Wellington, New Zealand 